\title{
Assessment of HIV/AIDS educational intervention on stigma reduction among nurses: a quasi-experimental study
}

\author{
John Mark Montoya Gutierrez \\ Department of Nursing, College of Applied Medical Sciences, Shaqra University, Kingdom of Saudi Arabia \\ E-mail: johnmark_gutierrez01@yahoo.com
}

\begin{abstract}
Background: In a setting where HIV/AIDS is highly endemic, nurses may respond to patients with increase fear of being at risk of acquiring HIV infection, decrease willingness to care, or both. The resulting stigmatization creates social barriers and discrimination between nurses and patients with HIV/AIDS.

Objectives: This study aimed to increase nurses' knowledge on HIV/AIDS and reduce HIV/AIDS-related stigma and discrimination in healthcare through HIV/AIDS educational intervention.

Methods: This quasi-experimental study was carried out in all nursing departments of Shaqra General Hospital from February to April 2014. Nurses $(n=58)$, meeting the eligibility criteria, were included in the sample and were non-randomly assigned in one-group pre-test and post-test using purposive sampling technique.

Results: The results of the pre- and post-intervention phases were compared using paired-sample $t$-test. Significant increase in nurses' knowledge was demonstrated following HIV/AIDS educational intervention $(p=0.000)$. HIV/AIDS educational intervention was significantly associated with less stigmatization for 'attitudes towards imposed measures' $(p=0.002)$. In pre-intervention phase, the greatest stigmatizing attitude was observed in statement concerning the need for screening all inpatients for HIV/AIDS. The assessment at preand post-intervention for 'comfortableness dealing with HIV/AIDS patients' did not differ significantly, indicating least stigmatizing attitude. However, a statistical significant difference $(p=0.044)$ on nurses' overall attitude towards patients with HIV/AIDS was detected between pre- and post-intervention assessment. Pooled mean revealed no observed discriminatory act among nurses at pre- and postintervention assessment.

Conclusion: The utilization of HIV/AIDS educational intervention improved nurses' theoretical knowledge about HIV/AIDS. Although nurses' knowledge on HIV/AIDS was improved significantly, their attitudes of blame or judgment as well as their comfortableness dealing with HIV/AIDS patients have not changed. The effectiveness of HIV/AIDS educational intervention in reducing HIV/AIDS-related discrimination as well as its impact on nurses' attitudes towards patients with HIV/AIDS warrants further investigation.
\end{abstract}

Keywords: Attitudes, Discrimination, Educational Intervention, HIV/AIDS, and Stigma.

\section{Introduction}

Since the outbreak of human immunodeficiency virus (HIV) infection and acquired immunodeficiency syndrome (AIDS) in the early 1980's, it is becoming difficult to ignore its gradual increase. The condition, which started locally in Africa, has become epidemic in most countries in the last thirty years. Recent developments in the field of medicine and nursing have led to a renewed interest in gaining and preventing efforts toward elimination commitments on HIV/AIDS; however, significant issues and challenges still remain. Along with the growth of HIV/AIDS patients, there is an increasing concern over the HIV-related stigma, discrimination, punitive laws and practices in healthcare as factors that fuel HIV/AIDS worldwide.

Stigma and discrimination among people living with HIV/AIDS (PLWHA) persist within many healthcare facilities. In his seminal work, 'Stigma and social identity: Notes on the management of spoiled identity,' Goffman (1963, p. 4) described stigma as a 'special kind of relationship between attribute and stereotype' that significantly discredits the former in the society. His idea is grounded in the concept of social identity that is based on estab lishing 'normal' and 'stigmatized' individual (Andrewin \& Chien 2008).

Statistically in 2013, the United Nations Agency for International Development (UNAIDS) report on global AIDS has estimated that 35.3 million people were living with HIV. In Saudi Arabia, the cases continue to have an HIV epidemic with more than 10,000 estimated cases. This number reflects the expansion of HIV/AIDS cases in the country.

The issue has grown in importance in the light of literature showing that $80 \%$ of the nurses' and $90 \%$ of the physicians' behavior with the HIV-positive individuals were discriminatory (Ullah 2011). HIV-infected patients are experiencing refusal of healthcare services and stigmatizing attitudes (i.e., attitudes of blame or judgment) from healthcare providers, nurses in particular The debate about the resulting stigmatization has gained prominence with means of arguing that patients with HIV/AIDS are one of the challenging issues in nursing that needs to be addressed. This process, if completely ignored, creates social barriers and discrimination between nurses and patients with HIV/AIDS.

In a setting where HIV infection and AIDS are highly endemic, nurses may respond to patients with increase fear of being at risk of acquiring HIV infection, decrease willingness to care, or both. 
The issues on social barriers, stigmatization, and acts of discrimination associated with the HIV/AIDS as well as the cultural sensitivity of the case have been controversial and much disputed subject among certain population. Such issues prevent PLWHA from seeking medical attention, voluntary HIV testing and counseling. Furthermore, differences by gender, race or ethnicity, education, income, disability, geographic location and sexual orientation play a significant role in access to healthcare services. In addition, social determinants of health like poverty, unequal access to health care, lack of education, stigma, and racism are linked to health disparities (CDC 2013).

Despite the safety and efficacy on the management of HIV/AIDS, patients suffer from serious major drawbacks. Similarly to the stigma report conducted by Herek et al. (2008), they have pointed out that HIV/AIDS is associated with high levels of stigma because of its perceived unalterable, degenerative, and fatal condition. Andrewin \& Chien (2008) stressed that this stigmatizing attitude towards PLWHA by significant portion of the public is a result of perceived immoral and voluntary actions. For instance, homosexual and promiscuous sex as well as sharing of infected needles among injection drug users (Adrewin \& Chien 2008) is considered forbidden in Islamic culture.

A number of research studies have reported the importance of effective HIV/AIDS policies and educational programs in reducing expressed and enacted stigma. In their study, Al-Marzou et al (2005) carried out an interventional study on a sample selected from paramedical students of health institutes and health colleges in Saudi Arabia. Based on results, their findings have a positive effect on students' knowledge and attitudes towards HIV/AIDS. Preference of confidentiality was also significantly increased among paramedical students following educational intervention. This indicates a need to understand the various perceptions of nurses that exists in healthcare facilities with vast cultures.

One of the most current discussions have elucidated the importance of educational efforts about universal precaution, ethical issues, and patient rights in order to reduce stigmatization and discrimination among patients with HIV/AIDS (Zeighami et al 2011). Despite significant program efforts, numerous studies have linked HIV-related stigma with delayed HIV testing, nondisclosure to partners, poor engagement with HIV services (Brou 2007, Bwirire 2008 \& Karim 2008) and ineffective HIV/AIDS policy and education program. Sengupta et al. (2011) suggest that future studies must focus on designing interventions with greater internal validity and validated HIV/AIDS stigma instruments. This study addresses if HIV/AIDS educational intervention is associated with stigma and discriminatory reduction in healthcare.

Moreover, nurses play a significant role in applying the learned theoretical knowledge directly to care and treatment of patient with HIV/AIDS. However, translating theoretical knowledge into nursing practice has received little attention because this requires a series of clinical transition in professional role practice. The provision of continuing educational training program is essential to help nurses expand their theoretical nursing knowledge regarding HIV/AIDS and to improve their clinical nursing judgment. The controversy about scientific evidence for nurses has raged unabated for decades. Although numbers of studies have investigated nurses' theoretical knowledge and attitudes about HIV/AIDS, transforming attitudes towards care and treatment of patients with HIV/AIDS remains to be a clinical challenge among nurses.

Investigating the recent evidence about stigmatization and discrimination among patients with HIV/AIDS as well as the insufficient evidence about the effectiveness of HIV/AIDS educational intervention in reducing HIV/AIDS-related stigma is a continuing concern that motivated this study to assess its effects among nurses. This study aimed to increase nurses' willingness to care, reduce fear of being at risk of acquiring HIV, and decrease stigmatization and discrimination in care and treatment of patients with HIV/AIDS by refining nurses' knowledge and attitudes about HIV/AIDS through educational training program. This study is designed to help nurses expand their theoretical knowledge and enhance their care practices for patients with HIV/AIDS.

\section{Methods}

\subsection{Design and procedures}

A quasi-experimental, one-group pre-test and post-test design was utilized. The study investigated the effects of educational intervention on nurses' knowledge about HIV/AIDS, as well as on nurses' stigmatizing attitudes and acts of discrimination towards PLWHA. The study is completely voluntary and has been approved by the hospital research ethics committee.

The study was divided into three phases: Pre-intervention phase assessed nurses' theoretical knowledge about HIV/AIDS using a valid 40-item self-administered survey questionnaire. Nurses' attitudes and discriminatory acts towards patients with HIV/AIDS were also determined. In intervention phase, the researcher conducted series of 90-minute educational training program regarding HIV/AIDS. Three educational sessions were held to ensure maximum attendance among participants assigned to all nursing shifts. The elements of the program covered HIV/AIDS updates, epidemiology, risk groups, etiology, immunology, courses and manifestations, transmission, universal precaution and prevention, ethical issues, patient rights, stigmatization model, and stigma reduction strategies using evidence-based approach. After the discussion, short forum to answer necessary clarifications from the participants was set. In post-intervention phase, same set of questionnaire is re-administered to eligible study participants.

\subsection{Study population and setting}

This study was carried out in Shaqra General Hospital (SGH) from February to April 2014. Nurses were recruited to estimate the effects of HIV/AIDS educational intervention in reducing healthcare-related stigma and discrimination. Eligibility criteria were used to screen study participants. Nurses, either both genders, who encountered patients with HIV/AIDS, have no formal HIV/AIDS training, were employed for 6 months or more, and currently working at the time of survey were considered eligible for the current study.

\subsection{Sampling}

A total of 58 of the 157 nurses were considered eligible for this study. Nurses, meeting the eligibility criteria, were included in the sample and were non-randomly assigned in one-group pre-test and post-test using a purposive sampling technique. To minimize selection bias, the researcher established similar baseline characteristics (inclusion criteria) among study participants, assigned them to one-group design, and determined differential lost to follow-up. All nurses completed the self-administered survey questionnaires at pre- and post-intervention phase and participated in HIV/AIDS educational intervention, yielding response and completion rates of $100 \%$.

\subsection{Data gathering instrument}

The researcher utilized a valid close-ended survey questionnaire. This instrument measures nurses' theoretical knowledge about HIV/AIDS, attitudes towards patients with HIV/AIDS and nurses' acts of discrimination. The questionnaire was developed by Andrewin and Chien (2008) as a tool for assessing stigmatization of patients with HIV/AIDS and was approved by three experts using content validity index. The researcher added slight modifications to the survey questionnaire to ensure content validity and cultural acceptability among study participants. Additional 33item instrument developed by Eckstein (1987) was used to measure nurses' HIV/AIDS knowledge. Prior to the use of the instrument, consent was obtained from these authors.

The questionnaire consisted of two parts. The first part included a survey letter to the study participants, indicating their consent. The second part consisted of different domains: (1) nurses' HIV/AIDS knowledge; (2) attitudes towards patients with HIV/AIDS; and (3) 
acts of discrimination by nurses. The outcome variables of this study were nurses' HIV/AIDS knowledge, stigmatizing attitudes towards patient with HIV/AIDS and discriminatory acts.

Nurses' HIV knowledge was assessed using forty-item questionnaire for which the overall frequency and percentage of accurate responses was calculated. Eckstein (1987) identified seven subcategories of knowledge indicators that evaluate participants' areas of strength and weakness. These include (1) agent (3 items), (2) immunology (3 items), (3) course and manifestations (5 items), (4) transmission (5 items), (5) incidence (2 items), (6) risk group (6 items), and (7) precaution and prevention (9 items). Adrewin and Chien also identified four subcategories of knowledge indicators. These include (1) transmission (2 items), (2) precaution and prevention (2 items), (3) incidence (1 item) and (4) risk group (1 item). Additional item (HIV/AIDS description) was added to make a total 40-item survey questionnaire measuring nurses' HIV/AIDS knowledge. The scale used for frequency and percentage of responses was 1 (not knowledgeable), 2 (slightly knowledgeable), 3 (knowledgeable), 4 (moderately knowledgeable) and 5 (highly knowledgeable).

In the domain of attitudes towards patients with HIV/AIDS, Andrewin \& Chien (2008), ranked the items in 'assessing stigmatizing attitudes' using five-point Likert-type scales. Based on their tool, responses to each statement ranged from 1 (strongly disagree) to 5 (strongly agree). The items 'assessing acts of discrimination' were similarly ranked with responses to those statements ranging from 1 (never) to 5 (all of the time). A score of 1 was regarded as not stigmatizing or discriminatory and 5 being most stigmatizing or discriminatory with a higher score thereof indicating a more stigmatizing attitude or a higher frequency of committing discriminatory act. The authors applied necessary reverse coding to allow consistent direction of association. Andrewin and Chien also conducted principal components factor analysis, utilizing variation max rotation with Kaiser normalization, a loading criterion of 0.6, and eigenvalues of one or greater. Based on the criteria, the authors measured the nine items that fit into three subscales: (1) attitudes toward imposed measures -4 items, Cronbach $\alpha 0.71$; (2) attitudes of blame/judgment -3 items, Cronbach $\alpha 0.60$; and (3) comfortableness in dealing with HIV/AIDS patients -2 items, Cronbach $\alpha 0.83$. The developers of this valid instrument summed and averaged the items to give stigmatizing scores for each subscale. Andrewin \& Chien also treated separately the five items used to assess discriminatory acts in the analysis.

Prior to distribution of the survey questionnaire, test-re-test method was used to ensure instrument reliability as well as to determine its cultural acceptability. Twenty nurses in a different hospital were recruited as a pilot sample. Spearman rho was used to determined level of reliability. The results of reliability test are displayed in Table 1.

\subsection{Data analysis}

Results were presented as frequencies and percentages, mean and standard deviations. A paired-sample $t$-test analysis was used to evaluate the results of the pre- and post-intervention phase. Statistical significance was set at $p<0.05$ (95\% confidence interval). All analyses were achieved using Statistical Package for the Social Sciences (SPSS) for Windows version 15.0.

\subsection{Ethical consideration}

This current study has been reviewed and approved by the hospital research ethics committee in SGH. The responses were treated with confidentiality, and the anonymity of the participants was assured while ensuring the freedom from harm after providing the discussion of the elements of disclosure of essential information. Participants were also ensured that this study is completely voluntary. Record keeping was maintained and all data were stored in secured place with only the researcher has access.

\section{Results}

\subsection{Effects on nurses' knowledge}

Nurses' theoretical knowledge about HIV/AIDS was determined by analyzing the frequency and percentage of correct responses. The results of the pre- and post-intervention phases were compared using paired-sample t-test. In pre-intervention phase, nurses are highly knowledgeable about disease description (100\%). However, their knowledge regarding agent, immunology, courses and manifestations, incidence and precaution and prevention was moderate, with $60.3 \%$ to $64.4 \%$ of respondents answered correctly. The results about knowledge in disease transmission $(53.7 \%)$ and risk groups $(47.8 \%)$ indicate that nurses are knowledgeable in these subcategories.

Significant increase in nurses' knowledge was demonstrated following HIV/AIDS educational intervention $(p=0.000)$. The mean overall number of frequency and percentage of correct responses in the post-intervention assessment was higher compared to preintervention assessment (30.6 [76.4\%] versus 23.7 [59.3\%]; $p=$ $0.000)$. Knowledge about HIV (50 [86.2\%]; $p=0.000)$, immunology (46.3 [79.9\%]; $p=0.000)$, courses and manifestations (47 [81\%]; $p=0.000)$, transmission, (42.7 [73.4\%]; $p=0.000)$, incidence (46 $[79.3 \%] ; p=0.000)$, risk groups (39.4 [67.9\%]; $p=$ $0.000)$, precaution and prevention (43.6 [75.2]; $p=0.000)$ improved significantly following HIV/AIDS educational intervention. A more detailed description of nurses' HIV/AIDS knowledge results is illustrated in Table 2 .

Table 1: Parts of Survey Questionnaire with Spearman Rho Value

\begin{tabular}{lllll}
\hline Part & No. of Items & Content & rho value & Description \\
\hline 1 & 40 & Nurses' HIV/AIDS knowledge & 0.76 & High reliability \\
2 & 9 & Attitudes towards patients with HIV/AIDS & 0.92 & Very high reliability \\
3 & 5 & Acts of discrimination by nurses. & 0.95 & Very high reliability \\
\hline
\end{tabular}

Table 2: Nurses' HIV/AIDS Knowledge; Pre-and Post-Intervention Phase

\begin{tabular}{|c|c|c|c|}
\hline \multirow{2}{*}{ Subcategories and items (Correct answer) } & \multicolumn{2}{|c|}{ Frequency and percentage of correct response } & \multirow[b]{2}{*}{$p$ value } \\
\hline & Pre-intervention $(\mathrm{n}=58 ; \%)$ & Post-intervention $(\mathrm{n}=58 ; \%)$ & \\
\hline Disease description & $58(100)$ & $56(96.6)$ & 0.159 \\
\hline 1) There's no known cure for HIV/AIDS (T). & $58(100)$ & $56(96.6)$ & 0.159 \\
\hline Agent & $37(63.8)$ & $50(86.2)$ & 0.000 \\
\hline 2) The incubation period for HIV/AIDS is $2-5$ weeks $(\mathrm{F})$. & $46(79.3)$ & $51(87.9)$ & 0.000 \\
\hline $\begin{array}{l}\text { 3) The HIV/AIDS virus is very difficult to kill with disinfectant in the environ- } \\
\text { ment (F). }\end{array}$ & $34(58.6)$ & $52(89.7)$ & 0.000 \\
\hline 4) HIV/AIDS is caused by a retrovirus known as HTLV-III/LAV (T). & $31(53.4)$ & $47(81)$ & 0.167 \\
\hline Immunology & $35(60.3)$ & $46.3(79.9)$ & 0.000 \\
\hline $\begin{array}{l}\text { 5) HIV/AIDS is characterized by a decrease in T lymphocytes, causing an im- } \\
\text { paired cellular immunity (T). }\end{array}$ & $51(87.9)$ & $49(84.5)$ & 0.419 \\
\hline 6) A person with antibody to the virus is protected against HIV/AIDS (F). & $34(58.6)$ & $50(86.2)$ & 0.000 \\
\hline $\begin{array}{l}\text { 7) An individual may be infected with the HIV/AIDS virus even if the test for an } \\
\text { antibody is negative (T). }\end{array}$ & $20(34.4)$ & $40(69)$ & 0.000 \\
\hline
\end{tabular}


8) Persons with HIV can be asymptomatic, but still infectious (T).

$35.6(61.4)$

$48(82.8)$

$44(75.9)$

10) One should suspect the diagnosis of HIV/AIDS in young persons who present with Kaposi's sarcoma (T).

11) Opportunistic infection (such as Candida esophagitis) in a previously healthy person is suggestive of HIV/AIDS (T).

12) The average length of time from the diagnosis of HIV/AIDS until death is 5 years $(\mathrm{F})$.

Transmission

13) HIV/AIDS has been transmitted to people receiving blood transfusion (T).

14) It is possible to transmit the virus to family members of a nurse providing care for persons with HIV/AIDS, even though the nurse is not infected (F).

15) HIV/AIDS can be transmitted by casual contact (F).

16) The risk of transmission of the HIV/AIDS virus during mouth-to-mouth resuscitation is extremely low (T).

17) One can contract HIV infection by sharing meals with an HIV-infected person (F).

18) HIV/AIDS has been transmitted to blood donors during blood transmission (F).

19) Most newborns born to HIV-positive women have HIV/AIDS infection at birth (F).

Incidence

20) There are many more people infected with HIV than actual AIDS (T).

21) Through sexual contact, gonorrhea is more likely transmitted than HIV virus (T).

22) Numerous cases of HIV/AIDS have been reported among nurses and midwives (T).

Risk groups

23) Intravenous drug abusers are considered to be at risk for contracting HIV/AIDS (T).

24) Members of the high-risk groups for HIV/AIDS are permitted to donate blood if they test negative for the antibody to the virus $(\mathrm{F})$

25) After needle stick injury with needle from an HIV-infected person, the chance of contracting HIV virus is less than $1 \%(\mathrm{~T})$.

26) Following an accidental needle stick, there is a greater likelihood of infection with hepatitis B virus than with HIV/AIDS (T).

27) The greatest risk of exposure to HIV/AIDS is caring for an incontinent patient with HIV/AIDS (F)

28) The risk of infection with HIV/AIDS among nurses is high (F).

29) The risk of infection with the HIV/AIDS virus after an accidental needle stick is high (F).

Precaution and prevention

30) Procedures for avoiding Hepatitis B and HIV infection are similar (T).

31) Heterosexual women do not get HIV/AIDS (F).

32) It is appropriate to use blood precautions on anyone known to be from HIV/AIDS high-risk group (such as a patients with hemophilia admitted for a tooth extraction) even though they do not have a diagnosis of HIV/AIDS (T).

33) The sexual partners of a person with HIV/AIDS should be blood precaution if hospitalized $(\mathrm{T})$.

34) Gloves are not necessary when handling the specimen of a patient with HIV/AIDS (F).

35) People with HIV/AIDS should have different waiting rooms before admission to the ward $(\mathrm{F})$.

36) To prevent accidental injury, contaminated needles should be recapped immediately after use on patients with HIV/AIDS (F)

37) People with HIV/AIDS should have separate bathroom/toilet facilities (F).

38) HIV/AIDS is highly contagious (F).

39) Gloves and gowns are required for any contact with patients with HIV/AIDS (F).

40) After needle stick injury with needle from an HIV-infected person, immediately gently expressing blood from the puncture site reduces the risk of contracting HIV infection (T)

Abbreviation: $\mathrm{T}$, true; F, false

Note: For each subcategory, items listed in order from highest to lowest percentage of correct response at pre-intervention phase

The scores for responses were as follows: "not knowledgeable" =1; "slightly knowledgeable" = 2; "knowledgeable" = 3; "moderately knowledgeable"

$=4$; "highly knowledgeable" $=5$

\subsection{Effects on nurses' attitude}

In pre-intervention phase, the greatest stigmatizing attitude (mean 3.24) was observed in statement concerning the need for screening all inpatients for HIV/AIDS. HIV/AIDS educational program was significantly associated with less stigmatization for attitudes towards imposed measures $(p=0.002)$. No significant differences were found on nurses' attitude of blame or judgment towards patients with HIV/AIDS. However, nurses' expressed stigma towards people who get AIDS from intravenous drug abuse improved significantly $(p=0.013)$ following HIV/AIDS educational intervention. In addition, the assessment at pre- and postintervention for comfortableness dealing with HIV/AIDS patients did not differ significantly, indicating least stigmatizing attitude.

\begin{tabular}{|c|c|}
\hline $47(81)$ & 0.000 \\
\hline $50(86.2)$ & 0.484 \\
\hline $50(86.2)$ & 0.001 \\
\hline $47(81)$ & 0.000 \\
\hline $46(79.3)$ & 0.000 \\
\hline $42(72.4)$ & 0.033 \\
\hline 42.7 (73.4) & 0.000 \\
\hline 45 (77.6) & 0.096 \\
\hline $46(79.3)$ & 0.000 \\
\hline $46(79.3)$ & 0.000 \\
\hline 45 (77.6) & 0.057 \\
\hline $43(74.1)$ & 0.004 \\
\hline $38(65.5)$ & 0.000 \\
\hline $36(62.1)$ & 0.001 \\
\hline $46(79.3)$ & 0.000 \\
\hline $51(87.9)$ & 0.133 \\
\hline $43(74.1)$ & 0.000 \\
\hline 44 (75.9) & 0.252 \\
\hline 39.4 (67.9) & 0.000 \\
\hline $49(84.5)$ & 0.260 \\
\hline $48(82.6)$ & 0.028 \\
\hline $31(53.4)$ & 0.209 \\
\hline $34(58.6)$ & 0.000 \\
\hline $40(69)$ & 0.019 \\
\hline $36(62.1)$ & 0.000 \\
\hline 38 (65.5) & 0.000 \\
\hline $43.6(75.2)$ & 0.000 \\
\hline $46(79.3)$ & 0.322 \\
\hline 49 (84.5) & 0.070 \\
\hline $44(75.9)$ & 0.011 \\
\hline $50(86.2)$ & 0.000 \\
\hline 45 (77.6) & 0.322 \\
\hline $48(82.6)$ & 0.532 \\
\hline $40(69)$ & 0.096 \\
\hline $\begin{array}{l}41(70.7) \\
44(75.9)\end{array}$ & $\begin{array}{l}0.034 \\
0.000\end{array}$ \\
\hline $36(62)$ & 0.000 \\
\hline $37(63.8)$ & 0.484 \\
\hline
\end{tabular}

$0(86.2)$

$(81)$

$42(72.4)$

45 (77.6)

$43(74.1)$

$8(65.5)$

$46(79.3)$

$1(87.9)$

44 (75.9)

0.000

0.028

0.019

0.000

0.322

0.000

0.532

0.034

0.000

0.484

))

Although no significant differences were detected on nurses' attitude of blame or judgment towards patients with HIV/AIDS and their comfortableness dealing with HIV/AIDS patients, pooled mean of nurses' overall attitude towards patients with HIV/AIDS revealed a statistical significant difference $(p=0.044)$ between pre- and post-intervention assessment. The results of nurses' attitudes towards patients with HIV/AIDS are displayed in Table 3.

\subsection{Effects on nurses' HIV/AIDS-related practices}

At pre-intervention phase, the highest acts of discrimination score was observed for the item, getting consent from the patient before testing his/her blood for HIV (mean, 2.36; SD [standard deviation] 1.76) followed by giving differential treatment to patients based on HIV status (mean, 1.86; SD, 0.89) and disclosing a patient's 
HIV status to a colleague who is not directly involved in the case management (mean, 1.84; SD, 1.39). For these items, a reduction of mean scores was observed following HIV/AIDS educational intervention; however, mean scores at pre- and post-intervention phase did not show significant results. Acts of discrimination towards patients with HIV did not differ significantly $(p=0.197)$ following HIV/AIDS educational intervention. Least discriminatory acts were observed at pre- and post-intervention phase. Pooled mean revealed no observed discriminatory act among nurses at pre- and post-intervention assessment (1.76 versus $1.79 ; p=$ 0.838). Detailed results of each item are presented in Table 4.

Table 3: Nurses' Attitudes towards Patients with HIV/AIDS; Pre-and Post-Intervention Phase

\begin{tabular}{|c|c|c|c|c|}
\hline \multirow{2}{*}{\multicolumn{2}{|c|}{ Subscales and items }} & \multicolumn{2}{|c|}{ Mean of Responses $(n=58)$} & \multirow[b]{2}{*}{$p$ value } \\
\hline & & Pre-intervention Mean (SD) & Post-intervention Mean (SD) & \\
\hline \multicolumn{2}{|c|}{ Attitudes of blame/judgment } & $2.7(1.22)$ & $2.68(1.42)$ & 0.871 \\
\hline & $\begin{array}{l}\text { I feel more sympathetic toward people who get AIDS from blood transfu- } \\
\text { sions than those who get it from intravenous drug abuse. }\end{array}$ & $3.3(1.34)$ & $2.95(1.47)$ & 0.013 \\
\hline 2) & $\begin{array}{l}\text { I feel that if a child contracts the HIV/AIDS virus from its mother through } \\
\text { mother-child or vertical transmission, the mother is to blame for the child's } \\
\text { disease. }\end{array}$ & $2.34(0.87)$ & $2.53(1.31)$ & 0.147 \\
\hline 3) & $\begin{array}{l}\text { I have little sympathy for people who get HIV/AIDS from sexual promiscui- } \\
\text { ty. }\end{array}$ & $2.47(1.19)$ & $2.57(1.45)$ & 0.490 \\
\hline \multicolumn{2}{|r|}{ Attitude towards imposed measures } & $3.06(1.26)$ & $2.67(1.29)$ & 0.002 \\
\hline & All patients admitted to the hospital should be HIV-tested. & $3.24(1.35)$ & $2.81(1.38)$ & 0.005 \\
\hline 5) & $\begin{array}{l}\text { Relatives/sexual partners of patients with HIV/AIDS should be notified of } \\
\text { the patient's status even without his/her consent. }\end{array}$ & $3.22(1.24)$ & $2.64(1.28)$ & 0.004 \\
\hline 6) & $\begin{array}{l}\text { Patients with HIV/AIDS should be cared for and treated in their own hospi- } \\
\text { tals and facilities, away from other patients who do not have HIV/AIDS }\end{array}$ & $2.7(1.25)$ & $2.55(1.34)$ & 0.124 \\
\hline & $\begin{array}{l}\text { A health professional with HIV/AIDS should not be working in any area of } \\
\text { health care that requires patient contact. }\end{array}$ & $3.02(1.15)$ & $2.67(1.18)$ & 0.044 \\
\hline \multicolumn{2}{|r|}{ Comfortableness dealing with HIV/AIDS patients } & $2.48(0.81)$ & $2.34(1.16)$ & 0.196 \\
\hline & I am comfortable putting a drip in someone who is showing signs of AIDS ${ }^{\cdot b}$ & $2.52(0.84)$ & $2.38(1.20)$ & 0.289 \\
\hline \multicolumn{2}{|c|}{ Overall mean (SD) } & $2.75(0.57)$ & $2.56(1.03)$ & 0.044 \\
\hline
\end{tabular}

"The scores for responses were as follows: "strongly disagree" $=1$; "disagree" $=2$; "no opinion" = 3; "agree" = 4; "strongly disagree" = 5.

${ }^{b}$ Reverse coding applied to these items when score allotted

$\mathrm{SD}$, standard deviation.

Table 4: Nurses' Acts of Discrimination towards Patients with HIV/AIDS; Pre-And Post-Intervention Phase

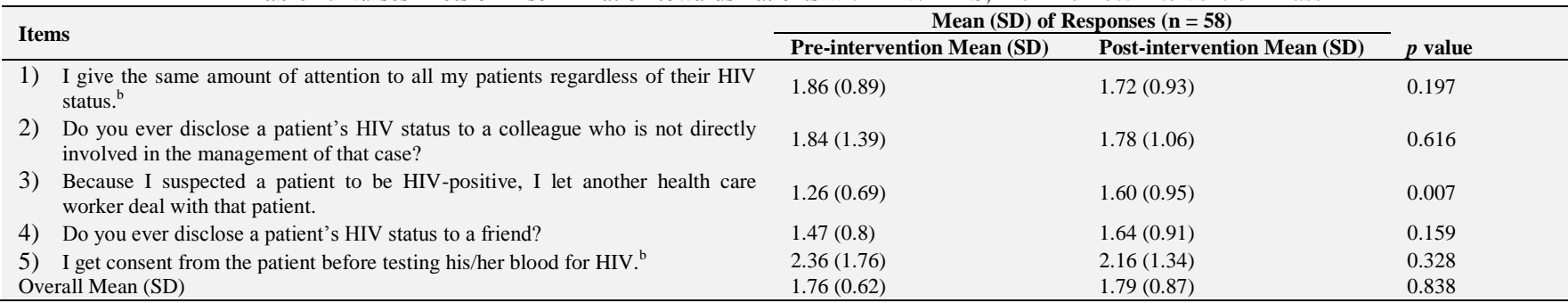

"The scores for responses were as follows: "never" =1; "a little of the time" $=2$; "some of the time" = 3; "most of the time" = 4; "all of the time" = 5.

${ }^{\mathrm{b}}$ Reverse coding applied to these items when score allotted.

$\mathrm{SD}$, standard deviation.

\section{Discussion}

To my knowledge, this study represents the largest series of registered nurses being evaluated in a quasi-experimental, one-group pre-test and post-test design to estimate the effects of HIV/AIDS educational intervention in reducing HIV/AIDS-related stigma and discrimination in Saudi Arabia. Data about nurses' knowledge on HIV/AIDS, attitudes towards HIV/AIDS patients and their acts of discrimination in Saudi Arabia are scarce. Such scarcity can be attributed to culture and sensitivity of the case. Unfortunately, insufficient research evidence has been published on the effects of HIV/AIDS educational intervention in reducing healthcare-related stigma and discrimination. Although previous research studies have reported the significance of HIV/AIDS educational intervention in reducing stigma and discrimination, designing interventions that pay greater attention to internal validity, use validated HIV/AIDS stigma instruments, and achieve both statistical and public health significance (Sengupta et al. 2011) are lacking. This current study offers a unique approach in evaluating the effects of HIV/AIDS educational intervention among nurses working in the different nursing departments in SGH. The researcher analyzed the effects of HIV/AIDS educational intervention on nurses' knowledge about HIV/AIDS, attitudes toward patients with HIV/AIDS and assessed their acts of discrimination. Based on the results, significant differences were observed in the domain of knowledge following HIV/AIDS educational intervention. While the attitudes towards imposed measures were statistically significant, the attitudes of blame or judgment towards patients with HIV/AIDS as well as the comfortableness dealing with HIV/AIDS patients were not significantly changed. Nurses' perceived attitude of blame or judgment towards patients with HIV/AIDS and comfortableness dealing with HIV/AIDS patients remained constant, regardless of HIV/AIDS educational intervention. The results further support the conclusion that the change in attitude of blame or judgment towards patients with HIV/AIDS and the comfortableness dealing with HIV/AIDS patients were not significant. Nurses' overall attitudes towards patients with HIV/AIDS have significantly reduced following HIV/AIDS educational intervention. Pooled mean also revealed no observed discriminatory act among nurses at pre- and post-intervention phase.

Healthcare providers, particular nurses, have different views and attitudes towards HIV-positive individuals. Hamama et al. (2014) explored nurses' job satisfaction and their attitudes towards PLWHA using self-reported questionnaires. Based on their findings, 69 nurses from the HIV/AIDS centers and 66 from the general hospital revealed that significant differences in nurses' attitudes towards PLWHA. Nurses in HIV/AIDS centers reported less avoidance than nurses in the general hospital. In the study of Ullah (2011), he revealed that health providers' views toward the HIVpositive individuals are not very much different from the general 
population. In fact, Ullah revealed that $80 \%$ of the nurses' and $90 \%$ of the physicians' behavior with the HIV-positive individuals were discriminatory. In his mixed method design, Hasan et al. (2012) reported that majority of the 238 participants reported facing negative reactions and discrimination from physicians, nurses, and laboratory technicians. The results of this current study are not consistent with those of earlier research suggesting that discriminatory acts are more prevalent among healthcare providers, particularly nurses. Although in this current study, expressed stigma is evident; enacted stigma is less likely being observed among nurses In this study, nurses working in general hospital revealed least discriminatory act prior educational intervention. However, their attitudes of blame or judgment and towards imposed measures were stigmatizing. For instance, nurses expressed stigma towards people who get AIDS from intravenous drug abuse and sexual promiscuity. Nurses also blame the mother if the child contracts the HIV/AIDS virus through mother-child or vertical transmission. Nurses are not comfortable working with health professional that has HIV/AIDS. In their epidemiological investigation, Al-Mazrou et al. (2005) mentioned that $77 \%$ of HIV-positive Saudis were male, with a male-to-female ratio of about 3 to 1 . Adults 15-49 years constituted $78 \%$ of cases, including $46 \%$ of cases infected through sexual activity. Al-Marzou also stressed that most cases (67\%) were registered in Jeddah, Riyadh and Dammam. In Muslim countries, HIV/AIDS is considered shameful, particularly in rural settings. Even discussions on this topic are frowned upon (Rai et al. 2007). In this study, nurses, working in rural setting, may have different views towards patients with HIV/AIDS because significant portion of the public to blame PLWHA for their illness, since the acquisition is perceived to be a result of immoral and voluntary actions, for example in homosexual and promiscuous sex and sharing of infected needles among injection drug users. Such AIDS-related shame was the best predictor of AIDS stigma in Saudi Arabia (Badahdah 2010).

The study has inherent limitations. First, in survey methods, respondents may not feel encouraged to provide accurate and honest answers or may not feel comfortable providing answers due to busy work schedules, culture as well as the sensitivity of the case. Moreover, in the survey instrument, respondents may interpret certain options differently because some options may represent different meanings to different respondents. In addition, results obtained about the level of knowledge acquired by nurses cannot be completely attributed to the educational intervention. The differences of the accuracy and completeness of the data obtained from pre-intervention phase to post-intervention phase may be influence by memory bias. Nevertheless, the researcher ensured that same set of questionnaires is being distributed at pre- and post-intervention phase on a six-week interval.

Another possible limitation of this study is the responses of nurses about their acts of discrimination. Their responses were not validated using other data gathering methodologies (i.e., observation or interview). Although there is no standardized psychometric tools used to assess nurses' discriminatory acts, this study trusts that the results obtained do subjectively assess nurses' acts of discrimination, as the statements in the survey questionnaire were based on a validated instrument with high reliability.

Since this was not a randomized study, other concomitant factors may have influenced the results. Nevertheless, the researcher ensured that nurses are not being treated differently. The HIV/AIDS educational intervention is made available to all eligible nurses and during this study, the enrollment of nurses remained constant from pre-to post-intervention phase, yielding response and completion rates of $100 \%$.

As this is a single-center study, the results obtained cannot be extrapolated to guide HIV/AIDS educational intervention as an effective intervention in reducing stigma and discrimination in healthcare associated with HIV/AIDS. Nevertheless, the effects of HIV/AIDS educational program should be evaluated positively.

\section{Conclusion}

This study aimed to increase nurses' knowledge on HIV/AIDS and reduce HIV/AIDS-related stigma and discrimination in healthcare by determining the effects of HIV/AIDS educational intervention. Based on the results, the utilization of HIV/AIDS educational intervention improved nurses' theoretical knowledge about HIV/AIDS. Although nurses' knowledge on HIV/AIDS was improved significantly, their attitudes of blame or judgment as well as their comfortableness dealing with HIV/AIDS patients have not changed. The current data were primarily generated among nurses who encountered patients with HIV/AIDS and have no formal HIV/AIDS training. These results are essential to refine program strategies for HIV/AIDS stigma reduction program. The results provide needs assessment to guide nurses in focusing on areas that are most crucial for effective service delivery. Nurses must use the results to improve their nursing practice in caring for PLWHA. Furthermore, the results are important to determine funding distribution for HIV/AIDS educational program for future evaluations.

This current study opens a new line for specifically determining the effects of HIV/AIDS educational intervention on nurses' HIV/AIDS knowledge, their attitudes towards PLWHA, and their acts of discrimination. Additional research is needed to examine the impact of HIV/AIDS educational intervention in reducing HIV/AIDS-related stigma and discrimination. Furthermore, the effectiveness of HIV/AIDS educational intervention in reducing HIV/AIDS-related discrimination as well as its impact on nurses' attitudes towards patients with HIV/AIDS warrants further investigation.

\section{Conflict of interest statement}

The author declares that there are no conflicts of interest.

\section{Acknowledgment}

The analyses and ideas contributed by the steering committee to this study are greatly appreciated. Special thanks to Jose Reinhard C. Laoingco, PhD, RN, my research advisor; to Alain Jason A. Generale, RN, MAN, PhDc; Roderick R. Ortega, RN, MSN; Keneth G. Bayani, MBA; and John Francis L. Faustorilla, DNS, $\mathrm{RN}$ for their constructive criticisms and critical revisions for important intellectual content. Thank you to Sheryl Ann V. Yap, RN, MANc; Russel S. Abalos, RN, MAN; and Allan L. Dueño, RN, MSN for their supervision and technical support. Invaluable contribution was also provided by Ramon Perley M. Pandaan, RN, MAN, LLB. My sincere gratitude extends to all the clinical staff nurses who participated in this study and to the facilitators who made this learning experience possible.

\section{References}

[1] Al-Mazrou YY, Abouzeid MS \& Al-Jeffri MH (2005), Impact of health education on knowledge and attitudes of Saudi paramedical students toward HIV/AIDS. Saudi Medical Journal, 26 (11), 17881795. DOI: 20050780

[2] Al-Mazrou YY, Al-Jeffri MH, Fidail AI, Al-Huzaim N, \& El-Gizouli SE (2005), HIV/AIDS epidemic features and trends in Saudi Arabia. Annals of Saudi Medicine, 25 (2): 100-104.

[3] Andrewin A \& Chien L (2008), Stigmatization of patients with HIV/AIDS among doctors and nurses in Belize. AIDS Patient Care and Sexually Transmitted Diseases 2(11), 897-906. DOI: 10.1089/apc.2007.0219 http://dx.doi.org/10.1089/apc.2007.0219.

[4] Badahdah AM (2010), Stigmatization of Persons with HIV/AIDS in Saudi Arabia. Journal of Transcultural Nursing, 21(4), 386-392. DOI: 10.1177/1043659609360873. http://dx.doi.org/10.1177/1043659609360873.

[5] Brou H et al. (2007), When do HIV-infected women disclose their HIV status to their male partner and why? A study in a PMTCT programme. Abidjan. Public Library of Science Medicine, 4(2), e342. 
DOI:

10.1371/journal.pmed.0040342

http://dx.doi.org/10.1371/journal.pmed.0040342.

[6] Bwirire LD et al. (2008), Reasons for loss to follow-up among mothers registered in a prevention-of-mother-to-child transmission program in Rural Malawi. Transaction of the Royal Society of Tropical Medicine and Hygiene, 102(12), 1195-1200. DOI 10.1016/j.trstmh.2008.04.002 http://dx.doi.org/10.1016/j.trstmh.2008.04.002.

[7] Center for Disease Control and Prevention (2013), Disparities in HIV/AIDS, viral hepatitis, STDs, and TB. Available at: http://www.cdc.gov/nchhstp/healthdisparities/ (Accessed 14 February 2014).

[8] Eckstein EC (1987), Knowledge and attitudes of nurses regarding patient with acquired immunodeficiency syndrome. A thesis presented to the Faculty of the Frances Payne Bolton School of Nursing, Case Western Reserve University.

[9] Goffman E (1963), Stigma and social identity: Notes on the management of spoiled identity. Prentice Hall, Inc., Englewood Cliffs, NJ, p.

[10]Hamama L, Tartakovsky E, Eroshina K, Patrakov E, Golubkova A, Bogushevich J, \& Shardina L (2014), Nurses' job satisfaction and attitudes towards people living with HIV/ AIDS in Russia. International Nursing Review, 61(1), 131-139. DOI: 10.1111/inr.12074 http://dx.doi.org/10.1111/inr.12074.

[11]Hasan MT, Khan NS, Akram O, Gomes TM, \& Rashid SF (2012), Experiences of discrimination among people living with HIV/AIDS in Bangladesh. Asia Journal of Public Health, 3(2), 44-52.

[12]Herek GM, Mitnick L, Burris S, Chesney M, Devine P, Thompson Fullilove M, Fullilove R, Gunther HC, Levi J, Michaels S, Novick A, Pryor J, Snyder M, \& Sweeney T (2008), Workshop report: AIDS and stigma: A conceptual framework and research agenda. AIDS Public Policy Journal, 16-25.

[13]Karim, QA et al. (2008), the influence of AIDS stigma and discrimination and social cohesion on HIV testing and willingness to disclose HIV in rural KwaZulu-Natal, South Africa. Global Public Health, 3(4), 351-365. http://dx.doi.org/10.1080/17441690802076496.

[14]Rai MA, Warraich HJ, Ali SH, \& Nerurkar VR (2007), HIV/AIDS in Pakistan: The battle begins. BioMed Central Journal; 1-3.

[15] Sengupta S, Banks B, Jonas D, Miles MS \& Smith GC (2011), HIV interventions to reduce HIV/AIDS stigma: A systematic review. AIDS and Behavior, 15(6), 1075-1087. http://dx.doi.org/10.1007/s10461010-9847-0.

[16]Ullah AKMA (2011), HIV/AIDS-related stigma and discrimination: A study of health care providers in Bangladesh. Journal of the Interna tional Association of Physicians in AIDS Care; 10 (2): 97-104. DOI 10.1177/1545109710381926. http://dx.doi.org/10.1177/1545109710381926.

[17]UNAIDS (2013), Global report: UNAIDS report on the global AIDS epidemic 2013. Available at: http://www.unaids.org/en/media/unaids/contentassets/documents/epid emiology/2013/gr2013/UNAIDS_Global_Report_2013_en.pdf (Accessed 15 March 2014)

[18]UNAIDS \& WHO (2008). 2007 Middle East and North Africa: AIDS epidemic update regional summary.

[19]Zeighami MS, Zeinali E, Esmaily H \& Nikbakht Nasrabadi A (2011), Fear of being at risk of acquiring HIV, willingness to care, and discrimination in care and treatment of AIDS patients among nurses. Scientific Journal of Iranian Blood Transfusion Organization, 8(3), 217228. 